\title{
PRIMERA CITA EN SUDAMÉRICA DE TRES ESPECIES ALÓCTONAS DE ÁFIDOS (HEMIPTERA, APHIDIDAE) RECOGIDOS EN LA ARGENTINA
}

\author{
J. Ortego ${ }^{1}$, M. P. Mier Durante ${ }^{2} \&$ J. M. Nieto Nafría ${ }^{3}$ \\ ${ }^{1}$ Instituto Nacional de Tecnología Agropecuaria (INTA), Estación Experimental Agropecuaria Mendoza. \\ San Martín 3853; 5507 Luján de Cuyo (Mendoza, Argentina). E-mail: ortegojaime@yahoo.com.ar. \\ ORCID iD: https://orcid.org/0000-0001-9738-7085 \\ 2 Departamento de Biodiversidad y Gestión Ambiental, Universidad de León. 24071 León (Spain). \\ E-mail: mpmied@unileon.es. ORCID iD: https://orcid.org/0000-0001-7834-1778 \\ ${ }^{3}$ Departamento de Biodiversidad y Gestión Ambiental, Universidad de León. 24071 León (Spain). \\ E-mail: jmnien@unileon.es. ORCID iD: https://orcid.org/0000-0001-6714-2415
}

\section{RESUMEN}

Se citan por primera vez en Sudamérica tres especies de áfidos (Hemiptera, Aphididae) a partir de colectas realizadas en las provincias argentinas de Mendoza, Río Negro y San Luis: Uroleucon taraxaci (Kaltenbach, 1843) relacionada con la adventicia Taraxacum officinale, y Siphonatrophia cupressi Swain, 1918 y Liosomaphis berberidis (Kaltenbach, 1843) que pueden afectar a plantas de jardinería. Se aportan datos morfológicos que las caracterizan y otros sobre su biología y distribución, que facilitarán el seguimiento de su posible dispersión en América del Sur.

Palabras clave: Especies alóctonas; pulgones; áfidos; Aphididae; América del Sur; Argentina.

\section{ABSTRACT}

First South-American record for three allochthonous aphid-species (Hemiptera, Aphididae) collected in Argentina.

Three species of aphids (Hemiptera, Aphididae) are recorded for the first time in South America from specimens collected in the Argentine provinces of Mendoza, Rio Negro and San Luis: Uroleucon taraxaci (Kaltenbach, 1843) related to the adventitious plant Taraxacum officinale, and Siphonatrophia cupressi Swain, 1918 and Liosomaphis berberidis (Kaltenbach, 1843) that can affect to garden plants. Morphological data that characterize each species and other data about their biology and distribution are provided, in order to facilitate the monitoring of the possible dispersion of these aphid-species in South America.

Key words: Allochthonous species; aphids; Aphididae; South America; Argentina

Recibido/Received: 28/02/2018; Aceptado/Accepted: 2/10/2018; Publicado en línea/Published online: 29/10/2018

Cómo citar este artículo/Citation: Ortego, J., Mier Durante, M. P. \& Nieto Nafría, J. M. 2018. Primera cita en Sudamérica de tres especies alóctonas de áfidos (Hemiptera, Aphididae) recogidos en la Argentina. Grael/sia, 74(2): e080. https://doi. org/10.3989/graellsia.2018.v74.205

Copyright: (C) 2018 SAM y CSIC. This is an open-access article distributed under the terms of the Creative Commons Attribution 4.0 International (CC BY 4.0) License. 


\section{Introducción}

Los pulgones o áfidos (Hemiptera, Sternorrhyncha, Aphididae) considerados en su conjunto, algo más de 5000 especies, están extendidos por todo el Mundo, con predominio en las regiones templadas y templado-frías, pero la mayoría de sus especies tienen áreas de distribución restringidas a alguna parte de los continentes. Su régimen alimenticio es sapívoro y la mayor parte de las especies están restringidas a vivir en una o unas pocas especies de plantas relacionadas. Sus ciclos vitales son más o menos complejos y una o varias de sus formas generacionales tienen una buena capacidad de dispersión natural. Cualquiera de ellas y también los huevos de invierno, que ponen las hembras ovíparas, pueden ser polizones indeseados en el transporte de plantas vivas.

En Argentina han sido citadas hasta el momento unas 250 especies, más que en cualquier otro de los países de Sudamérica (elaboración propia).

En este país y también en el conjunto de Sudamérica, la mayoría de las especies inventariadas son alóctonas, fundamentalmente de origen paleártico, habiéndose revelado algunas como invasoras y varias como dañinas y causantes, potenciales o reales, de plagas o de la transmisión de virus, como es el caso de Myzus persicae (Sulzer, 1776), Schizaphis graminum (Rondani, 1852) o Macrosiphum euphorbiae (Thomas, 1878), entre otras.

Continuando los trabajos de inventario de la afidofauna sudamericana, y especialmente argentina, que desde hace varias décadas vienen desarrollando los autores y algunos otros entomólogos, se citan por vez primera en América del Sur tres especies de pulgones pertenecientes a la tribu Macrosiphini (Aphididae); una de ellas es propia de una planta adventicia que también es alóctona y dos se desarrollan sobre plantas de jardinería de los géneros Berberis y Cupressus.

El seguimiento de la dispersión de las especies alóctonas no se debe descuidar y para facilitarlo con respecto a las tres especies que se tratan aquí, se proporcionan caracteres que permiten una identificación presuntiva de especímenes que se observen en plantas de especies que los puedan hospedar. Esa identificación debería ser corroborada con un estudio taxonómico más detallado en el laboratorio y en su caso refrendada por un especialista.

\section{Material y métodos}

Como es habitual en trabajos de esta naturaleza, en un primer momento los especímenes recogidos se conservaron en etanol al $70 \%$ y posteriormente se montaron en preparaciones microscópicas, recurriendo para su identificación a caracteres tanto cualitativos como cuantitativos, siguiendo en todo los procedimientos expuestos por Nieto Nafría \& Mier Durante (1998). En la parte dedicada a cada una de las tres especies se detalla el material estudiado, que se conserva en la colección de J. Ortego, en Mendoza (Argentina).

En Aphid Species File (Favret, 2018), obra en línea que se actualiza continuamente, se puede obtener información sobre sinonimias y combinaciones de las tres especies que se tratan.

\section{Resultados}

Uroleucon (Uromelan) taraxaci (Kaltenbach, 1843)

Material estudiado (en todo caso hembras vivíparas ápteras). Mendoza: Malargüe, 12-III-2015; San Carlos, 13-III-2015; Ciudad, 25-V-2015, Godoy Cruz, 28-V-2015. San Luis: Coronel Pringles, 23-VI-2016.

El género Uroleucon Mordvilko, 1914 con cerca de 240 especies es el más amplio de los géneros de Macrosiphini. Es el género de áfidos por detrás de Aphis Linnaeus, 1758 con más especies citadas en Argentina y en toda Sudamérica, 14 y 21 respectivamente, la mayoría nativas sudamericanas (elaboración propia).

La mayor parte de las especies de Uroleucon se hospedan en plantas de la familia Asteraceae, otras muchas en plantas de Campanulaceae, y algunas en plantas de otras familias, siendo destacable Uroleucon (Lambersius) adesmiae Mier Durante \& Ortego, 2008 , porque es una especie que está ligada exclusivamente a plantas de la familia Fabaceae (Mier Durante \& Ortego, 2008; Blackman \& Eastop, 2018).

En general son pulgones de tamaño grande, con antenas más largas que el cuerpo, largas patas, cornículos cilíndricos y también muy largos, y cola frecuentemente larga también.

Está admitido que el género se divide en seis subgéneros, tres de los cuales son monoespecíficos y no tienen representación en Sudamérica. El subgénero Uromelan Mordvilko, 1914 se caracteriza por la presencia de una fuerte pigmentación simultáneamente en las coxas y en la cola, similar a la que presentan los cornículos. Incluye 61 especies (Favret, 2018); en Sudamérica y también en Argentina se conocían hasta ahora tres de ellas: $U$. aeneum Hille Ris Lambers, 1939, U. compositae (Theobald, 1915) y U. jaceae (Linnaeus, 1758), todas alóctonas.

Las vivíparas de $U$. taraxaci son grandes, de 2,5 a casi 4,0 $\mathrm{mm}$ de longitud corporal, como también lo son las ovíparas, mientras que los machos son notoriamente menores, casi enanos, como es habitual en el género. En vida presentan color cobrizo brillante más o menos oscuro, con manchas dorso-abdominales aún más oscuras que se corresponden con los numerosos escleritos setíferos (véanse fotos en influentialpoints. com [InfluentialPoints, 2018]).

Una parte de estos caracteres son compartidos con U. compositae, también citada sobre Taraxacum (véase Blackman \& Eastop, 2018). Poder discernir la 
pertenencia de vivíparas ápteras recogidas sobre plantas de Taraxacum a una u otra de estas dos especies no es muy complicado: en $U$. taraxaci (véanse fotos en influentialpoints.com) las antenas y las tibias son enteramente negras, la cola es relativamente corta, relativamente ancha y finaliza en punta bastante aguda, y de haber escleritos postcorniculares no son más grandes que los numerosos escleritos precorniculares, mientras que en $U$. compositae las antenas y las tibias son pálidas en parte, la cola es larga y lanceolada y hay escleritos postcorniculares grandes.

Uroleucon taraxaci vive en la porción de las hojas que está en contacto con el suelo, preferentemente cerca de la raíz o incluso al comienzo de ésta (véanse fotos en influentialpoints.com [InfluentialPoints, 2018]), con frecuencia formando grupos. Típicamente es holocíclica con machos ápteros. En Argentina no se han encontrado todavía formas sexuadas.

Uroleucon taraxaci ha sido citada en varios países de Europa y de Asia Central y en Norteamérica y se la cita por lo tanto por vez primera en América del Sur.

En Argentina se la ha encontrado hasta el momento en dos provincias, Mendoza y San Luis, aunque se ha buscado en otros lugares. Es posible que la especie haya entrado en Argentina hace no mucho tiempo y es posible que pueda extenderse por gran parte de América del Sur, ya que su planta hospedadora es conocida en todos las provincias argentinas, así como en amplias zonas de Chile, en Uruguay y en el sur de Paraguay y de Brasil (Instituto de Botánica Darwinion, 2018).

\section{Liosomaphis berberidis (Kaltenbach, 1843)}

Material estudiado. Mendoza: Guaymallén, 1-V-2012, vivíparas ápteras; Luján de Cuyo, 26-X-2012, viv. ápt.; 05-V-2012 y 09-IV-2013, viv. ápt. y aladas; 20-V-2014, viv. ápt. y al. y ovíparas; Godoy Cruz, 17-VIII-2014, viv. ápt. y al. Río Negro: San Carlos de Bariloche, 17-IV-2012, viv. ápt. En todo caso sobre plantas de hojas rojas, posiblemente de Berberis thunbergii var atropurpurea.

Liosomaphis berberidis es una de las cuatro especies de Liosomaphis Walker, 1868. Es conocida en buena parte del paleártico desde el occidente europeo hasta la India, mientras que las otras tres especies tienen distribuciones asiáticas más o menos restringidas al este de Asia Central. Blackman \& Eastop (2018), relacionan 38 especies de Berberis y una de Mahonia como hospedadoras de la especie. Algunas de esas 38 son de origen sudamericano, pero se han citado como hospedadoras de L. berberidis en países fuera de América del Sur (Holman, 2009).

Las vivíparas ápteras adultas de $L$. berberidis son pequeñas, 1,1 a 2,3 $\mathrm{mm}$ de longitud corporal según Blackman \& Eastop (2018), ovaliformes, con cornículos y cola evidentes aunque pálidos (a veces los cornículos algo tostados) y de colores que varían desde el verde amarillento al naranja rojizo o parduzco, pasando por diversas tonalidades del amarillo. Los adultos de las restantes formas generacionales son de tamaños semejantes y algo más esbeltos. Las vivíparas aladas y los machos (que son alados) tienen abdómenes coloreados como los de las ápteras, y cabeza y tórax más oscuros, y además los machos tienen manchas oscuras pareadas en el abdomen. Las ovíparas presentan un color amarillo pajizo a naranja oscuro o parduzco, coloración que es consecuencia de la presencia de amplios escleritos torácicos y abdominales segmentarios bien pigmentados (pueden verse fotos en biodiversidadvirtual.org [Asociación Fotografía y Biodiversidad, 2018]).

Con una lupa de pocos aumentos es perceptible que las antenas son nítidamente más cortas que el cuerpo, que los cornículos son hinchados, con un corto pedúnculo (parte proximal cilíndrica) y que la cola siempre es alargada y en las vivíparas casi cilíndrica. Con mayores aumentos (microscopio estereoscópico, en el laboratorio) se puede apreciar en las ápteras que la frente es sinuosa, con un tubérculo medial protuberante y en las aladas que hay sensorios secundarios en los artejos III a V de la antena.

En América del Sur sobre plantas de ese género se han citado también Aphis patagonica Blanchard, 1944 y A. berberidorum Ortego \& Mier Durante, 1997. Los especímenes de ambas especies son de color oscuro, negro, gris o violáceo, y presentan cornículos más o menos cilíndricos o troncocónico-alargados. Algunas especies polífagas de los géneros Aphis Linnaeus, 1758, Aulacorthum Mordvilko, 1914, Macrosiphum Passerini, 1860 y Myzus Passerini, 1860 [concretamente M. persicae (Sulzer, 1776)] conocidas en Argentina han sido citadas en otras partes del Mundo sobre especies de Berberis, y quizás pudieran colonizar en Argentina plantas de ese género. La única de ellas con cornículos hinchados es $M$. persicae, que es reconocible por la forma de la frente, con dos grandes y convergentes tubérculos laterales y por la coloración, que casi siempre es verde, y sus alados además por presentar una placa dorsoadbdominal.

Liosomaphis berberidis suele ocupar el envés de las hojas habitualmente sin formar grupos densos, puede también colonizar los brotes, el haz de las hojas y en su momento flores y frutos; en otoño, cuando los arbustos pierden la mayoría de sus hojas, se pueden observar sobre ramitas semileñosas.

Las vivíparas aladas parecen ser poco frecuentes y las ovíparas fueron observadas en pleno otoño; no se capturaron machos pero es posible que la especie pueda cerrar el ciclo, aunque también es posible pensar que algunas vivíparas puedan pasar el invierno en plantas situadas en emplazamientos bien protegidos.

La revisión permanente y reiterada de plantas de especies nativas de Berberis en lugares próximos a 
los de las colectas arrojó resultados negativos, por lo que podría pensarse que $L$. berberidis se limita en Argentina a colonizar plantas de jardín o escapadas de cultivo. No se ha encontrado en localidades de Jujuy, Salta, Tucumán, Catamarca, Córdoba, San Luis, San Juan, Neuquén y La Rioja, a pesar de las numerosas búsquedas efectuadas, por lo que se puede afirmar que aún no está muy difundida.

\section{Siphonatrophia cupressi (Swain, 1918)}

Material estudiado. Jujuy: Humahuaca, Uquía, Cupressus sempervirens, 11-III-2017, vivíparas ápteras. Mendoza: Luján de Cuyo, Estación Experimental Agropecuaria (INTA), C. macrocarpa, 12-V-2011, viv. ápt.; 20-VIII-2011, viv. ápt. y aladas; 25-VII-2015, viv. ápt. San Luis: Junín, Merlo, C. sempervirens, 22-VI-2016, viv. áp.

Siphonatrophia cupressi es la única especie del subgénero Siphonatrophia Swain, 1918, y la única del género que está ligada a plantas de los géneros Cupressus, Juniperus y Widdringtonia (Cupressaceae). Es conocida en América del Norte y Central, llegando hasta Costa Rica; se ha introducido en España, Francia e Italia, en Argelia y Túnez y en el Japón (Laamari et al., 2016; Ben Halima-Kamel \& Mdellel, 2017; Blackman \& Eastop, 2018).

Las vivíparas ápteras son pequeñas, 1,0 a 1,7 mm de longitud corporal según Blackman \& Eastop y casi hemisféricas, debido a que tienen la parte ventral muy plana y el dorso muy abombado. En vida tienen un color verde semejante a la parte de la planta sobre la que viven (Fig. 1). Tienen antenas más cortas que el cuerpo y patas también cortas, cola triangular y unas dos veces más larga que ancha en la base, y cornículos inapreciables a simple vista, troncocónicos, bajos y estrechos. Las vivíparas aladas, las ovíparas y los machos, que pueden ser ápteros o alados, tienen un aspecto semejante a las ápteras. La medial de las alas anteriores puede tener dos o tres ramas finales.

Las hembras vivíparas ápteras de esta especie no parasitadas son crípticas con la planta, sobre todo cipreses (Fig. 1A) y difíciles de apreciar cuando se encuentran en grupos poco numerosos; una vez localizadas, su identificación a simple vista es factible por esa peculiar forma del cuerpo (Fig. 1B) que es muy diferente de la que presentan la mayoría de pulgones $\mathrm{y}$ en todo caso aquellos que pueden vivir sobre cipreses: 1) los del género Cinara Curtis, 1835, que pueden tener color verdoso, son gruesos, entre ovoidales y esféricos, pero no abombados y tienen patas largas; 2) los de Illinoia morrisoni (Swain, 1918), que tienen color verde claro, son alargados con antenas, patas y cornículos largos; y 3) los de Aphis craccivora (Koch, 1854), especie que es conocida en Argentina sobre otras plantas, son de color negro brillante, ovoidales y con patas relativamente largas. Además las ápteras de todas ellas son ágiles y rápidas.

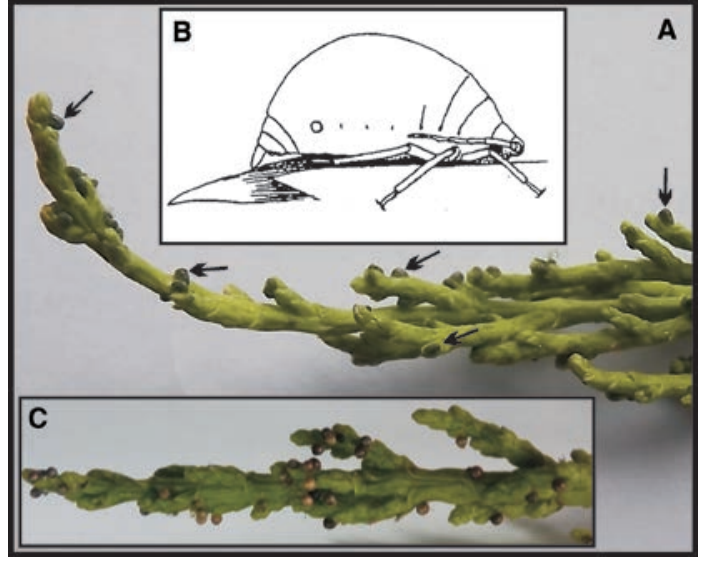

Fig. 1.- Siphonatrophia cupressi: A) hembras vivíparas ápteras sobre una ramita de ciprés en Luján de Cuyo (Argentina) [fotografía de J. Ortego], las flechas señalan algunas vivíparas ápteras; B) dibujo de una vivípara áptera de $S$. gravida (Knowlton, 1928) - sinónimo posterior de S. cupressi- sobre una hoja de Juniperus scopulorum [Palmer, 1952: pág. 225, fig. 264 (en parte)]; C) momias de hembras vivíparas ápteras sobre una ramita de ciprés en Luján de Cuyo (Argentina) [fotografía de J. Ortego].

Fig. 1.- Siphonatrophia cupressi: A) apterous viviparous females on a twig of cypress in Lujan de Cuyo (Argentina) [photograph by J. Ortego], arrows show some apterous viviparae; B) drawing of an apterous viviparae of $S$. gravida (Knowlton, 1928) -junior synonym of $S$. cupressi on a leave of Juniperus scopulorum [Palmer, 1952: pág. 225, fig. 264 (in part)]. C) mummies of apterous viviparous females on a twig of cypress in Luján de Cuyo (Argentina) [photograph by J. Ortego].

En los lugares de captura se observaron ejemplares dispersos hasta el punto de que en San Luis y en Jujuy se recogieron batiendo ramas, siempre acompañados por ejemplares de I. morrisoni, especie incorporada a la afidofauna conocida de Argentina hace unos pocos años (Mier Durante et al., 2010). Las búsquedas que de esta especie se han realizado durante los últimos años en cipreses en otras provincias argentinas (Córdoba, La Rioja, Neuquén, Salta, San Juan, Tucumán) y en otros países sudamericanos (Bolivia, Chile, Colombia), han resultado infructuosas.

En Estados Unidos la especie se comporta holocíclicamente, con sexuados en octubre o noviembre (Palmer, 1952), equivalente a abril y mayo del hemisferio Sur. En Argentina no se han localizado ni ovíparas ni machos y además las capturas de vivíparas en Mendoza, se realizaron durante la estación fría, desde mayo (fines del otoño) hasta agosto (mitad del invierno) y las reiteradas prospecciones en otros momentos del año e incluso en el mismo árbol, fueron infructuosas. Esto contrasta con el comportamiento de la especie en Europa y Japón, donde pulula desde finales del invierno hasta finales del otoño (Rabasse et al., 2005; Bella \& Squarcini, 2009; Sano \& Yoshitomi, 2012; Lumbierres et al., 2015). 
En casi todas las colectas se encontraron numerosos individuos parasitados por microhimenópteros, cuyas momias son fáciles de reconocer pues son de color pardo (Fig. 1C). Los adultos que fueron emergiendo fueron en todos los casos de Lysiphlebus testaceipes (Cresson, 1880) (Hym., Braconidae, Aphidiinae). Una relación idéntica ha sido observada en España y en Argelia (Lumbierres et al., 2015; Laamari et al., 2016).

\section{Agradecimientos}

Nuestro agradecimiento a Sara I. López Ciruelos (Servicio de Microscopia de la Universidad de León) por la edición de la figura 1 .

\section{Referencias}

Asociación Fotografía y Biodiversidad, 2018. Insectarium virtual. Liosomaphis berberidis. Disponible en http://www.biodiversidadvirtual.org/insectarium/ Liosomaphis-berberidis-(Kaltenbach-1843)-cat14033. html. Consultado: febrero 2018.

Bella, S. \& Squarcini, M., 2009. Si espande la diffusione in Italia dell'afide neartico del cipresso Siphonatrophia cupressi (Swain 1918). Bollettino di Zoologia Agraria e di Bachicoltura, 41: 61-64.

Ben Halima-Kamel, M. \& Mdellel, L., 2017. Présence de Siphonatrophia cupressi (Hemiptera: Aphididae) en Tunisie. OEPP EPPO Bulletin, 47(3): 549-550. https:// doi.org/10.1111/epp.12405

Blackman, R.L. \& V.F. Eastop, 2018. Aphids on World's plants. An online identification and information guide. Disponible en http://www.aphidsonworldsplants.info. Consultado: enero de 2018.

Favret, C., 2018. Aphids species file. Version 5.0/5.0. Disponible en aphid.speciesfile.org. Consultado: enero 2018.

Holman, J., 2009. Host Plant Catalog of Aphids. Palaearctic Region. Spinger Science + Busines Media, B.V. $6+$ $1216 \mathrm{pp}$.

Instituto de Botánica Darwinion, 2018. Catálogo de plantas vasculares de Flora del Cono Sur. Disponible en
http://www2.darwin.edu.ar/Proyectos/FloraArgentina/ fa.htm. Consultado: enero de 2018.

InfluentialPoints. 2018. InfluentialPoints - Services for ecologists, medics and veterinarians. Aphids identifications, characteristics of genera. Disponible en http:// influentialpoints.com/Gallery/Aphid_genera.htm. Consultado: febrero 2018.

Laamari, M., Aggoun, H., Starý, P. \& Coeur d'acier, A. 2016. First report of Siphonatrophia cupressi and its parasitoids in Algeria and a first record for Africa. OEPP EPPO Bulletin, 46 (1): 122-124. https://doi. org/10.1111/epp.12284

Lumbierres, B., Pérez Hidalgo, N., Starý, P. \& Pons, X. 2015. First record of the invasive Siphonatrophia cupressi (Swain) (Aphididae: Aphidini: Aphidina) in the Iberian Peninsula. Redia, 98, 141-144. Disponible en https://journals-crea.4science.it/index.php/redia/article/ view/1173. Consultado: febrero 2018.

Mier Durante, M.P. \& Ortego, J. 2008. A new species of Uroleucon (Hemiptera: Aphididae) living on Adesmia (Fabaceae). Florida Entomologist, 91(3): 367-371. https://doi.org/10.1653/0015-4040(2008)91\% 5B367:ANSOUH\%5D2.0.CO;2

Mier Durante, M.P., Ortego, J. \& Nieto Nafría, J.M., 2010. Primera cita en Argentina de tres especies de pulgones dendrófilos y nuevos datos sobre Neuquenaphis (Hemiptera Aphididae). Boletín de la Asociación Española de Entomología, 34(3-4): 335-341.

Nieto Nafría, J.M. \& Mier Durante, M.P. 1998. Hemiptera Aphididae I. In: M.A. Ramos et al. (Eds.). Fauna Ibérica, volumen 11. Museo Nacional de Ciencias Naturales, CSIC. Madrid. 424 pp.

Palmer, M.A., 1952. Aphids of the Rocky Mountain Region. The Thomas Say Foundation. Denver. 452 pp.

Rabasse, J.M., Coceano, P.G. \& Barbagallo, S. 2005. On the presence in France and North Italy of Siphonatrophia cupressi (Homoptera, Aphididae), a new aphid of North American origin living on Cupressaceae. Bollettino di Zoologia Agraria e di Bachicoltura, 37: 77-83.

Sano, M. \& Yoshitomi, H. 2012. First record of Siphonatrophia cupressi (Hemiptera, Aphididae) from Japan. Japanese Journal of Systematic Entomology, 18: 101-103. 